\title{
Controlling coherent and incoherent spin dynamics by steering the photoinduced energy flow
}

\author{
D. Bossini, ${ }^{1, *}$ A. M. Kalashnikova, ${ }^{2}$ R. V. Pisarev, ${ }^{2}$ Th. Rasing, ${ }^{1}$ and A. V. Kimel ${ }^{1}$ \\ ${ }^{1}$ Institute for Molecules and Materials, Radboud University Nijmegen, Heyendaalseweg 135, Nijmegen, The Netherlands \\ ${ }^{2}$ A. F. Ioffe Physical-Technical Institute, Russian Academy of Sciences, 194021 St. Petersburg, Russia \\ (Received 14 November 2013; revised manuscript received 28 January 2014; published 19 February 2014)
}

\begin{abstract}
We present a femtosecond spectroscopic magneto-optical investigation of the coherent and incoherent spin dynamics in the antiferromagnetic dielectric $\mathrm{KNiF}_{3}$. The pathways of the photoinduced energy flow to spins were controlled by tuning the pump photon energy. In particular, we demonstrate that laser pulses, with photon energy tuned to a nearly-zero-absorption region, excite the spin system without any signatures of heating of electrons or phonons. In this regime the ultrafast excitation of coherent spin waves is followed by a gradual increase of the spin temperature solely due to decoherence of the laser-generated magnons, as revealed by our simultaneous measurement of both the transversal and the longitudinal component of the spin dynamics.
\end{abstract}

DOI: 10.1103/PhysRevB.89.060405

PACS number(s): 78.20.Ls, 75.30.Ds, 75.50.Ee, 78.47.-p

In the rapidly developing area of spintronics, antiferromagnets are playing an increasingly important role [1,2]. However, the conventional way to control spins with the help of an external magnetic field [3] is not effective in antiferromagnets, because of the strong exchange field between the sublattices. This obstacle has ignited an intense search for an ultrafast and energy efficient control of antiferromagnet spins. Although coherent magnons can be generated by 100 fs laser pulses via impulsive stimulated Raman scattering (ISRS) [4-6], this mechanism is believed to be intrinsically inefficient, because of the unavoidable release of energy to the electrons and phonons [7]. The heating of electrons and phonons by laser radiation is a direct consequence of the absorption of light in the material. This process heavily dominates the photoinduced spin dynamics in metals [8-11]. In contrast, in magnetic dielectrics, absorption is much lower, which allows the excitation of spin systems via ISRS by near-infrared laser pulses. However, each successful demonstration of the ultrafast generation of coherent magnons via ISRS has been realized in magnetic oxides $[5,6,12,13]$, in which absorption is not negligible in most of this spectral range. This has led to the fundamental questions: How does optical absorption affect the ultrafast laser-induced spin dynamics? Can femtosecond optical laser pulses generate magnetic excitations in the absence of any absorption? If this is the case, which spin dynamics could be observed in this regime?

In this Rapid Communication we report our experimental study on the role of optical absorption in the laser-induced coherent and incoherent spin dynamics. In particular, we demonstrate that when the photon energy of the pump pulses is tuned to the transparency region, the laser-induced spin dynamics does not reveal any signatures of heating of electrons or phonons, as if a selective optical excitation of the spin system is achieved.

The dielectric antiferromagnet $\mathrm{KNiF}_{3}$ is an excellent candidate to address the aforementioned issues. It is characterized by a region of nearly zero absorption in the optical range, as shown in Fig. 1. This is related to a wide energy band gap ( $E_{g} \approx 6.2 \mathrm{eV}[14]$ ) which distinguishes this material

*d.bossini@ science.ru.nl from $3 d$ transition-metal magnetic oxides, whose energy gap is typically $E_{g} \approx 3-4 \mathrm{eV}[15,16]$. All the absorption bands visible in the spectrum are due to relatively weak phononassisted localized $d$ - $d$ transitions [14,17]. Of interest for our purpose, in the 1.5-3 eV range these features alternate with a transparency window around $2 \mathrm{eV}$, where the absorption coefficient is nearly zero. $\mathrm{KNiF}_{3}$ has a perovskite type crystal structure (point group $\mathrm{m3m}$ ) [18]. Two equivalent $\mathrm{Ni}^{2+}$ sublattices are antiferromagnetically coupled below the Néel temperature $T_{\mathrm{N}}=246 \mathrm{~K}$ [19]. This material is known as a Heisenberg antiferromagnet because of its very weak cubic magnetic anisotropy. The positive sign of the anisotropy constant determines the alignment of spins along the [001], [010], and [100] axes [20]. The magnetic structure is described in terms of the antiferromagnetic vector, defined as $\vec{L}=$ $\vec{M}_{1}-\vec{M}_{2}$, where $\left|\vec{M}_{1}\right|=\left|\vec{M}_{2}\right|=M_{0}$ are the magnetizations of the two sublattices and $M_{0}$ is their saturation magnetization.

We performed a series of magneto-optical pump-probe experiments with a setup similar to the one used in Ref. [21]. A detailed description is provided in Ref. [22]. The photon energy was tuned within the range of $1.5-3 \mathrm{eV}$, either to one of the absorption bands or to the transparency window, as shown in Fig. 1. We probed changes in the ellipticity of the probe beam, which in our experimental configuration (see the inset in Fig. 1) represent variations of the magnetic linear birefringence (MLB). MLB is the difference in the refractive indices experienced by light polarized along and perpendicularly to the direction of the spins. This magneto-optical effect is a quadratic function of the spin moment, being described by the symmetric part of the dielectric permittivity tensor [23,24] $\epsilon_{i j}^{(S)}=\epsilon_{j i}^{(S)}$. Thus, in an Heisenberg antiferromagnet, MLB is proportional to $L^{2}$. Measuring transient MLB allows us to detect simultaneously both the transversal and the longitudinal dynamics of $\vec{L}$, due to the contributions to the dielectric permittivity tensor proportional to $L_{z} L_{y}$ and $L_{z}^{2}$, assuming $\vec{L}$ directed along the $\hat{z}$ axis. In contrast, in earlier investigations of laser-induced spin dynamics in compensated antiferromagnets $[6,7,25]$, the transient magneto-optical Faraday rotation was measured. Since in the ground state the magnetization is zero, the Faraday rotation vanishes. A transient net magnetic moment proportional to $\gamma \vec{L} \times \partial \vec{L} / \partial t$ appears in the nonequilibrium state [26]. Therefore the transient Faraday 


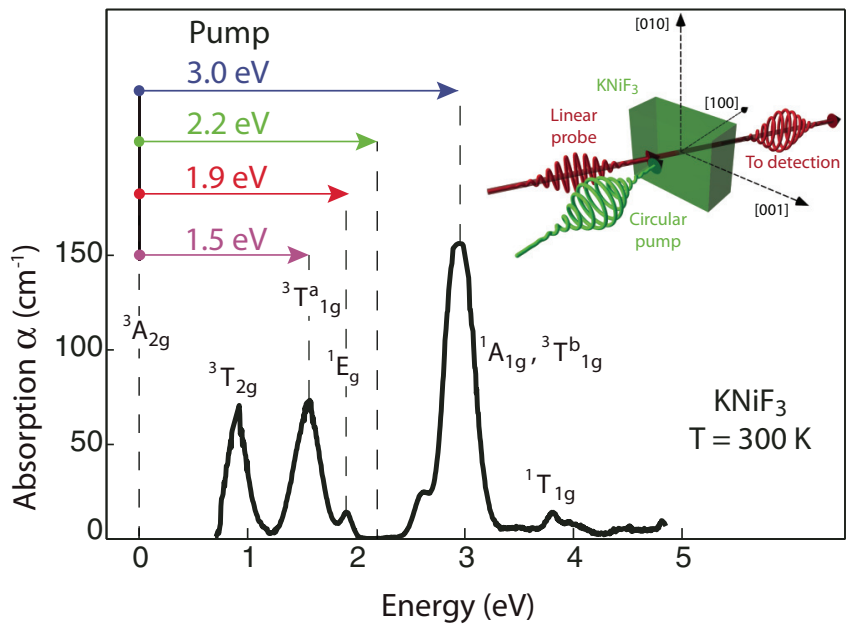

FIG. 1. (Color online) Spectral dependence of the absorption coefficient in $\mathrm{KNiF}_{3}$ at room temperature. The levels corresponding to the absorption bands and the ground state ${ }^{3} A_{2 g}$ are also shown. In the range $2-2.4 \mathrm{eV}$ a nearly-zero-absorption region is observed. The excitation photon energies used in the pump-probe experiment are indicated. In the inset, we report schematically the experimental geometry. We measured variations in the ellipticity of the probe polarization, due to magnetic linear birefringence.

effect gives access to the transversal, but not to the longitudinal dynamics of $\vec{L}$.

In order to demonstrate the excitation and detection of both the transversal and the longitudinal dynamics of $\vec{L}$ in $\mathrm{KNiF}_{3}$, we measured the transient MLB triggered by circularly polarized pump pulses resonant with the $1.5 \mathrm{eV}$ absorption band (see Fig. 1). Oscillations at a frequency of $v \approx 90 \mathrm{GHz}$ are visible in the first 80 ps superimposed on a slowly varying background described by the parameter $B$ [see Fig. 2(a)]. The temperature and pump polarization dependences of the oscillatory dynamics [22] allow us to ascribe it to the antiferromagnetic mode of coherent spin precession, resulting in transversal dynamics of $\vec{L}$ triggered via impulsive stimulated Raman scattering (ISRS). The inset of Fig. 2(a) shows the temperature dependence of the parameter $B$. The signal disappears approaching $T_{N}$, confirming its magnetic character. From this we conclude that the incoherent increasing response represents the longitudinal dynamics of $\vec{L}$. The diagram shown in Fig. 2(b) demonstrates the laser-induced processes leading to the observed spin dynamics. While a fraction of photons in the pump pulse participates in the generation of the coherent magnons via ISRS [27,28], another fraction of photons is absorbed, leading to a population of the upper localized $3 d$ band. These excited electrons relax on a time scale of about 1 ps via nonradiative processes, governed by electron-phonon coupling. As the temperature of the phonons $T_{\mathrm{ph}}$ increases, the excess energy is exchanged with the spin system on the $\approx 100$ ps time scale due to magnon-phonon coupling, resulting in an increase of the magnon temperature $T_{\mathrm{M}}$. The longitudinal incoherent dynamics is pump polarization independent [22], supporting our conclusion that it is caused by the energy flow (heating) to the magnetic system.

Aiming to reveal how the absorption affects the photoinduced spin dynamics, we performed measurements tuning the
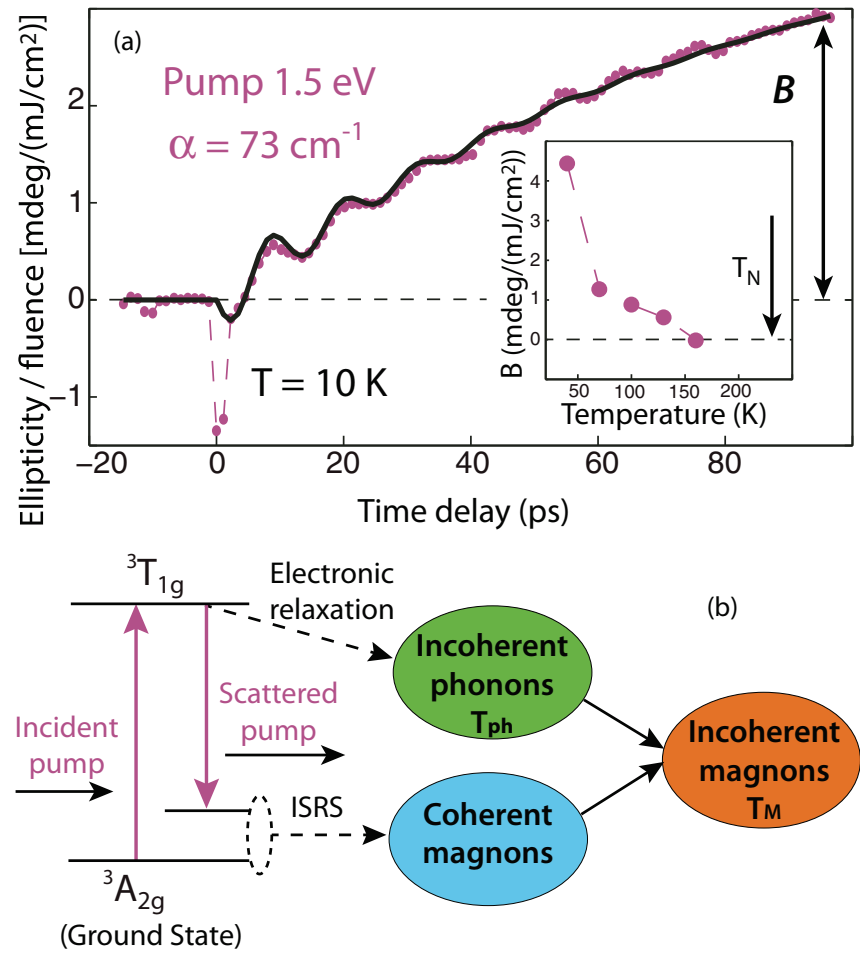

FIG. 2. (Color online) (a) Photoinduced changes in the ellipticity of the probe polarization as a function of the pump-probe delay. The data are normalized on the pump fluence, which was approximately $12 \mathrm{~mJ} / \mathrm{cm}^{2}$. The central photon energies of the pump and probe pulses were 1.5 and $1.9 \mathrm{eV}$, respectively. The temperature was $10 \mathrm{~K}$. The fit to the data was performed with the function reported in Eq. (1). The absorption coefficient corresponding to the pump photon energy is shown. The increase of the background is characterized by the parameter $B$ [see Eq. (1)]. In the inset we report the temperature dependence of the incoherent dynamics, measured with pump and probe photon energies set to $1.5 \mathrm{eV}$. The dashed line is a guide to the eye. (b) Diagram showing the energy flow to the spin system in the case of excitation resonant with an absorption band (see the main text).

pump photon energy to 3.0 and $1.9 \mathrm{eV}$ [see Fig. 3(a)]. For a quantitative comparison of the results, we fitted the time dependences with the following function:

$$
f(t)=\theta(t)\left[A \sin (2 \pi v t) e^{\left(-t / \tau_{d}\right)}+B\left(1-e^{\left(-t / \tau_{r}\right)}\right)\right],
$$

where $\theta(t)$ is a Heaviside function. The first and second terms in Eq. (1) describe the transversal and longitudinal dynamics of $\vec{L}$, respectively. The value of the frequency $v$ was taken from the Fourier transform of the data. The decoherence and characteristic rise time $\left(\tau_{d}\right.$ and $\left.\tau_{r}\right)$ and the amplitudes $(A$ and $B$ ) were fitting parameters. Their values are plotted in Figs. 3(b) and 3(c) as a function of the absorption coefficient. It is important to note that $B$ gradually increases with absorption in the range above $\alpha \approx 14 \mathrm{~cm}^{-1}$. The spectral dependence of the precession amplitude $A$ (not shown here) is less pronounced. We note that the decoherence time $\tau_{d}$ varies as a function of the absorption coefficient. We ascribe this behavior to the broadening of the magnon line, which depends on the inhomogeneous excitation caused by the optical absorption of 

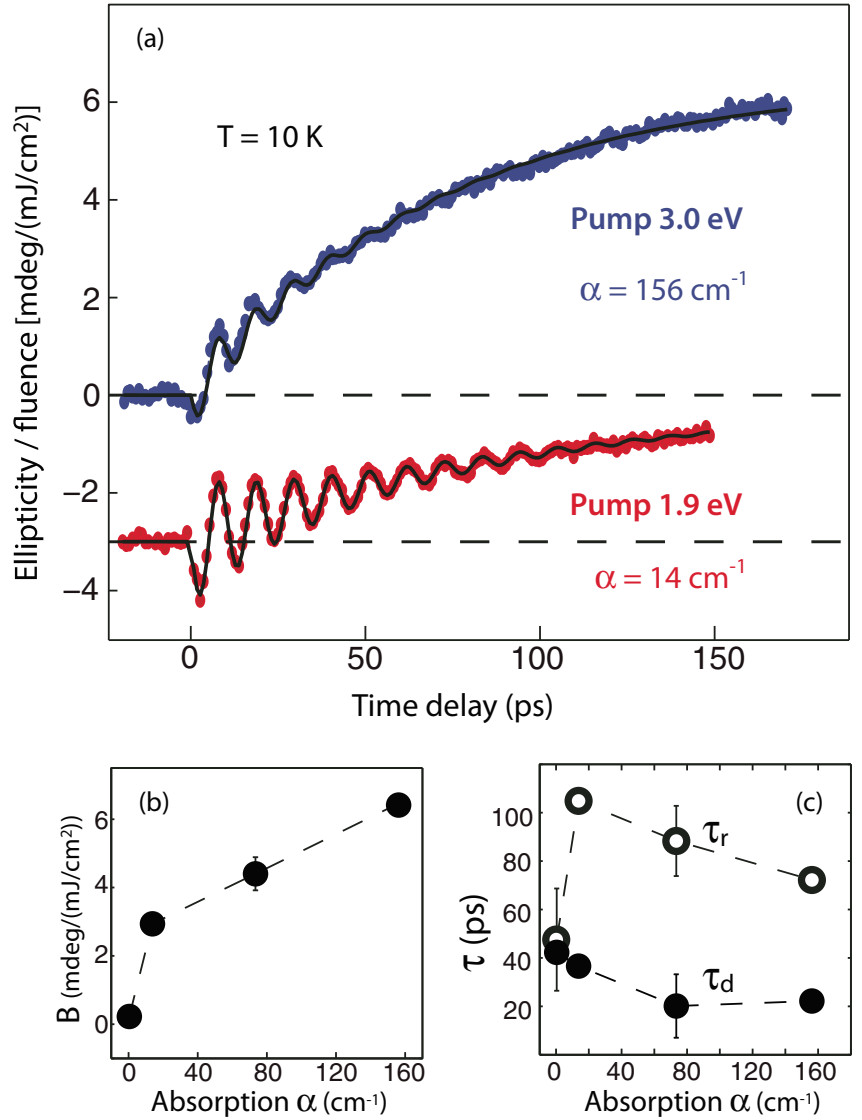

FIG. 3. (Color online) (a) Photoinduced changes in the ellipticity of the probe polarization as a function of the pump-probe delay obtained with the pump photon energy tuned to $3.0 \mathrm{eV}$ (blue dots) and $1.9 \mathrm{eV}$ (red dots). The probe photon energy was always $1.5 \mathrm{eV}$. The values of the absorption coefficient of the sample corresponding to these pump photon energies are reported. Solid lines are fits to the data using Eq. (1). (b) Amplitude $B$ of the incoherent dynamics obtained from the fit as a function of the absorption. A sharp decrease is observed for $\alpha \approx 0$. The dashed line is a guide to the eye. (c) Characteristic rise time of the incoherent dynamics (open dots) and decoherence time (solid dots) obtained from the fit as a function of the absorption. Note the abrupt decrease of $\tau_{r}$ at $\alpha \approx 0$. The dashed lines are guides to the eye. The error bars in (b) and (c) account for the standard deviation of the fit.

the pump beam. Moreover, Fig. 3(c) shows that the higher the absorption, the smaller is $\tau_{r}$.

The above described trends of both $B$ and $\tau_{r}$ break if we turn the light-matter interaction into the nearly-zero-absorption regime $\alpha \approx 0$. Figure 4(a) shows that setting the energy of the pump photons to $2.2 \mathrm{eV}$ induces a dramatic change in the incoherent response, while the amplitude of the oscillations is barely affected. The longitudinal laser-induced dynamics does not completely vanish despite the nearly zero absorption. Remarkably, the best fit to the data is obtained for $\tau_{r} \approx \tau_{d}$.

Such a consistency for the characteristic times [see Fig. 3(c)] distinguishes sharply the dynamics excited in nearlyzero-absorption conditions from the results obtained with other excitation energies. This suggests that the mechanisms dominating the longitudinal dynamics of $\vec{L}$ in these two regimes are different.
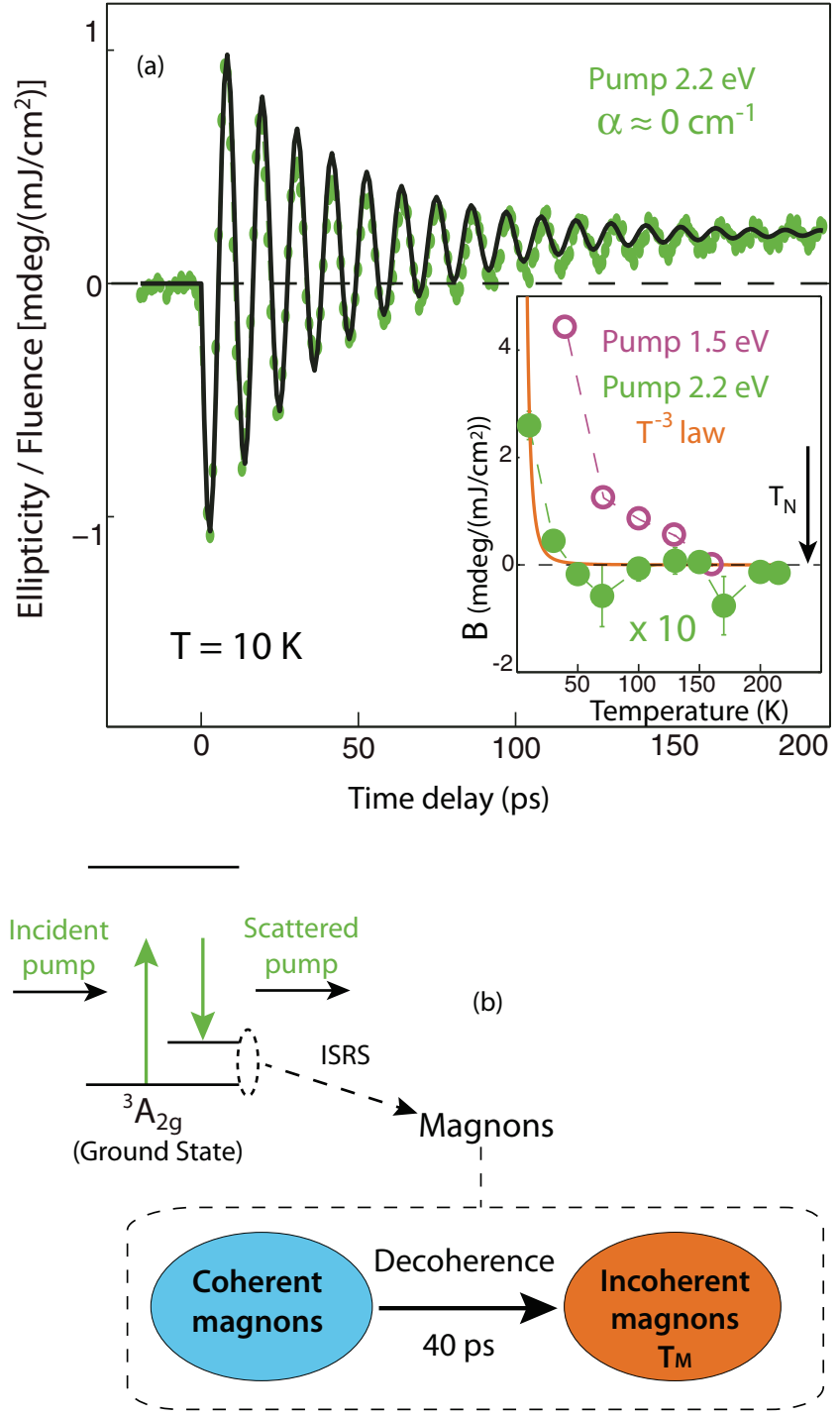

FIG. 4. (Color online) (a) Photoinduced changes in the ellipticity of the probe polarization as a function of the pump-probe delay. The fit to the data is performed using Eq. (1) (see the main text). In the inset we report the temperature dependence of the amplitude of the incoherent dynamics. Two experimental curves are reported: measurement performed with a pump photon energy of $1.5 \mathrm{eV}$ (open magenta dots) and $2.2 \mathrm{eV}$ (solid green dots, multiplied by a factor of 10 for the sake of comparison). The probe photon energy was $1.5 \mathrm{eV}$ in both cases. The two trends are fundamentally different, since the value of $B$ for the $2.2 \mathrm{eV}$ photon energy vanishes significantly faster than in the case of the $1.5 \mathrm{eV}$ stimulus. Data are normalized on the pump fluence. The error bars account for the standard deviation of the fit. The orange curve is a plot of the $T^{-3}$ law. (b) Diagram showing the energy flow to the spin system in case of excitation energy tuned to the nearly-zero-absorption regime (see the main text).

Let us now consider the laser-induced processes occurring when the medium is transparent for the pump pulse, as shown in Fig. 4(b). Since no real electronic transitions are excited, the energy deposited by the pump laser pulses into the medium is entirely stored in the magnetic ensemble, via ISRS. The lasergenerated coherent magnons scatter with a characteristic time $\tau_{d} \approx 40 \mathrm{ps}$, bringing the ensemble of these quasiparticles to decoherence. As a result, the spin temperature increases, traced 
by the longitudinal dynamics of the antiferromagnetic vector, with the very same characteristic time $\tau_{d}=\tau_{r} \approx 40 \mathrm{ps}$. This equality proves that, by tuning the energy of the pump photons to the nearly-zero-absorption spectral range, we achieved a regime in which the light-induced spin dynamics does not reveal any signatures of heated electrons or phonons, as if a selective excitation of spins only occurred. Even better, we can formulate a criterion allowing one to determine whether traces of optical absorption are present in the spin dynamics. This condition, which we call the zero-absorption criterion, is simply $\tau_{d}=\tau_{r}$.

Seeking further confirmation of this idea, we measured the temperature dependence of the longitudinal dynamics excited by 1.5 and $2.2 \mathrm{eV}$ pump pulses. A variation of the spin temperature $\Delta T_{\mathrm{M}}$ per unit volume is determined by the magnetic specific heat through the relation $W=C_{m} \Delta T_{\mathrm{M}}$, where $W$ is the energy delivered by the laser pulses to the spins via ISRS. The specific heat of an Heisenberg antiferromagnet follows the $C_{m} \propto T^{3}$ law [29], and consequently $\Delta T_{\mathrm{M}} \propto T^{-3}$. We plot this trend in the inset next to our temperature dependent experimental results. Let us underline that a fit to the nearly-zero-absorption regime data is not possible, given the low amount of statistically significant data points. The plot of the $T^{-3}$ law is aimed at a qualitative comparison, which provides a further confirmation of the different nature of the two regimes. The consistency between the plotted curve and the data obtained by pumping the material in the transparency window supports our interpretation. In this regime the heating of the spin system is uniquely due to decoherence of the light-induced ensemble of coherent magnons.

We also note that the laser-induced transversal spin dynamics in the nondissipative regime can be successfully modeled within the framework developed in Refs. [26,30-32]. The heating of the phonons and subsequent phonon-magnon energy transfer are not taken into account in this model. The effect of light on spins is expressed in terms of an effective magnetic field $\vec{H}_{\text {eff }}$, which we derive in Ref. [22]. Defining the unit vector $\vec{l} \equiv\left(\vec{M}_{1}-\vec{M}_{2}\right) / 2 M_{0}$, we can describe an antiferromagnet in terms of the following Lagrangian $[6,26]$ :

$$
\mathcal{L}(\vec{l})=\frac{\hbar}{2 \gamma H_{E}}\left(\frac{\partial \vec{l}}{\partial t}\right)^{2}-\mathcal{W}(\vec{l})-\frac{\hbar}{H_{E}}\left[\vec{H}_{\mathrm{eff}} \cdot\left(\vec{l} \times \frac{\partial \vec{l}}{\partial t}\right)\right],
$$

where $\gamma$ is the gyromagnetic ratio, $H_{E}$ is the exchange field, and $\mathcal{W}(\vec{l})$ is the anisotropy energy. The effective field $\vec{H}_{\text {eff }}$ is considered homogeneous over the thickness of the sample $(\approx 340 \mu \mathrm{m})$ and its duration is assumed to be equal to that of the laser pulses, i.e., $\approx 100$ fs. Only in the zero-absorption regime are these approximations well founded. The outcome of the theoretical model was used for a procedure alternative to the fit shown in Fig. 4(a), providing excellent agreement with the data [22].

Finally, we would like to note that an energy efficient ultrafast spin excitation has been believed to be accessible exclusively employing picosecond $\mathrm{THz}$ pulses [7]. Our results demonstrate that a proper choice of the photon energy allows for the achievement of a spin dynamics regime determined by a light-magnon interaction and subsequent relaxation as if phonons and electrons were not excited by light at all. We can draw this conclusion because, contrary to Refs. [6,7,25], we monitored simultaneously both the transversal and the longitudinal spin dynamics by measuring variations of MLB. Given the nonresonant nature of the coupling between light and spins in the ISRS process, the frequency range of the excitations accessible with this technique is intrinsically much broader. In our experimental setup ( $2.2 \mathrm{eV}$ pump energy) the excitation range amounts to $0-14 \mathrm{THz}$, which becomes 0-28 THz when the energy of the pump is tuned to $1.5 \mathrm{eV}$.

In conclusion, we disclose a regime of ultrafast spin dynamics in antiferromagnets in which no traces of heated electrons or phonons are present. Our experiments demonstrate the possibility to steer the energy flow to coherent and incoherent magnetic excitations by choosing the wavelength of the pump beam. This observation is possible only by measuring both the transversal and the longitudinal dynamics of the antiferromagnetic vector. The most significant outcome of our work is the formulation of the zero-absorption criterion, which allows one to determine whether spin dynamics reveals any traces of optical absorption.

The authors thank A. van Roij and A. J. Toonen for technical support. This research was partially supported by de Nederlandse Organisatie voor Wetenschappelijk Onderzoek (NWO), de Stichting voor Fundamenteel Onderzoek der Materie (FOM), the European Union's Seventh Framework Program (FP7/2007-2013) Grants No. NMP3-LA-2010246102 (IFOX), No. 280555 (Go-Fast), and No. 214810 (FANTOMAS), the European Research Council under the European Union's Seventh Framework Program (FP7/2007-2013)/ERC Grant Agreement No. 257280 (Femtomagnetism), as well as support from the Russian Ministry for Education and Science (Grant No. 14.B37.21.0899) and the Russian Foundation for Basic Research (Grant No. 12-02-31508). A.M.K. and R.V.P. acknowledge support from the Russian Government (Grant No. 14.B25.0031.25). A.M.K. acknowledges support from the Russian Foundation for Basic Research (Grant No. 12-02-31508).
[1] B. G. Park, J. Wunderlich, X. Martí, V. Holý, Y. Kurosaki, M. Yamada, H. Yamamoto, A. Nishide, J. Hayakawa, H. Takahashi, A. B. Shick, and T. Jungwirth, Nat. Mater. 10, 347 (2011).

[2] A. B. Shick, S. Khmelevskyi, O. N. Mryasov, J. Wunderlich, and T. Jungwirth, Phys. Rev. B 81, 212409 (2010).

[3] J. Stöhr and H. C. Siegmann, Magnetism From Fundamentals to Nanoscale Dynamics (Springer, Berlin, 2006).
[4] A. V. Kimel, A. Kirilyuk, P. A. Usachev, R. V. Pisarev, A. M. Balbashov, and Th. Rasing, Nature (London) 435, 655 (2005).

[5] A. Kirilyuk, A. V. Kimel, and Th. Rasing, Rev. Mod. Phys. 82, 2731 (2010).

[6] T. Satoh, S.-J. Cho, R. Iida, T. Shimura, K. Kuroda, H. Ueda, Y. Ueda, B. A. Ivanov, F. Nori, and M. Fiebig, Phys. Rev. Lett. 105, 077402 (2010). 
[7] T. Kampfrath, A. Sell, G. Klatt, A. Pashkin, S. Mährlein, T. Dekorsy, M. Wolf, M. Fiebig, A. Leitenstorfer, and R. Huber, Nat. Photonics 5, 31 (2010).

[8] E. Beaurepaire, J. C. Merle, A. Daunois, and J. Y. Bigot, Phys. Rev. Lett. 76, 4250 (1996).

[9] M. van Kampen, C. Jozsa, J. T. Kohlhepp, P. LeClair, L. Lagae, W. J. M. de Jonge, and B. Koopmans, Phys. Rev. Lett. 88, 227201 (2002).

[10] I. Radu, K. Vahaplar, C. Stamm, T. Kachel, N. Pontius, H. A. Dürr, T. A. Ostler, J. Barker, R. F. L. Evans, R. W. Chantrell, A. Tsukamoto, A. Itoh, A. Kirilyuk, Th. Rasing, and A. V. Kimel, Nature (London) 472, 205 (2011)

[11] J. H. Mentink, J. Hellsvik, D. V. Afanasiev, B. A. Ivanov, A. Kirilyuk, A. V. Kimel, O. Eriksson, M. I. Katsnelson, and Th. Rasing, Phys. Rev. Lett. 108, 057202 (2012).

[12] T. Satoh, Y. Terui, R. Moriya, B. A. Ivanov, K. Ando, E. Saitoh, T. Shimura, and K. Kuroda, Nat. Photonics 6, 662 (2012).

[13] R. Iida, T. Satoh, T. Shimura, K. Kuroda, B. A. Ivanov, Y. Tokunaga, and Y. Tokura, Phys. Rev. B 84, 064402 (2011).

[14] N. Mironova-Ulmane, V. Skvortsova, A. Kuzmin, and I. Sildos, Ferroelectrics 258, 177 (2001).

[15] R. V. Pisarev, A. S. Moskvin, A. M. Kalashnikova, and Th. Rasing, Phys. Rev. B 79, 235128 (2009).

[16] C. Rödl, F. Fuchs, J. Furthmüller, and F. Bechstedt, Phys. Rev. B 79, 235114 (2009).

[17] J. Ferguson, H. J. Guggenheim, and D. L. Wood, J. Chem. Phys. 40, 822 (1964).

[18] Y. Ivanov, E. A. Zhurova, V. V. Zhurov, K. Tanaka, and V. Tsirelson, Acta Crystallogr., Sect. B 55, 923 (1999).
[19] J. Nouet, A. Zarembowitch, R. V. Pisarev, J. Ferré, and M. Lecomte, Appl. Phys. Lett. 21, 161 (1972).

[20] L. Landau and E. Lifshitz, Electrodynamics of Continuous Media (Pergamon, New York, 1981).

[21] A. H. M. Reid, A. V. Kimel, A. Kirilyuk, J. F. Gregg, and Th. Rasing, Phys. Rev. Lett. 105, 107402 (2010).

[22] See Supplemental Material at http://link.aps.org/supplemental/ 10.1103/PhysRevB.89.060405 for the description of the setup, data as a function of the temperature and polarization. Details of the sigma model and the alternative fit procedure are also reported herein.

[23] J. Ferré and G. A. Gehring, Rep. Prog. Phys. 47, 513 (1984).

[24] G. A. Smolenskii, R. V. Pisarev, and I. G. Sinii, Sov. Phys. Usp. 18, 410 (1976).

[25] M. Takahara, H. Jinn, S. Wakabayashi, T. Moriyasu, and T. Kohmoto, Phys. Rev. B 86, 094301 (2012).

[26] A. F. Andreev and V. I. Marchenko, Sov. Phys. Usp. 23, 21 (1980).

[27] A. M. Kalashnikova, A. V. Kimel, R. V. Pisarev, V. N. Gridnev, A. Kirilyuk, and Th. Rasing, Phys. Rev. Lett. 99, 167205 (2007).

[28] A. M. Kalashnikova, A. V. Kimel, R. V. Pisarev, V. N. Gridnev, P. A. Usachev, A. Kirilyuk, and Th. Rasing, Phys. Rev. B 78 104301 (2008).

[29] L. Landau and E. Lifshitz, Statistical Physics, Part 2 (Pergamon, New York, 1980).

[30] V. G. Bar'yakhtar, B. A. Ivanov, and M. V. Chetkin, Sov. Phys. Usp. 146, 417 (1985).

[31] V. G. Bar'yakhtar and B. A. Ivanov, Fiz. Nizk. Temp. 5, 759 (1979).

[32] A. K. Zvezdin, JETP Lett. 29, 553 (1979). 